\title{
A Successful OSS Adaptation and Integration in an e-Learning Platform: TEC Digital
}

\author{
Mario Chacon-Rivas ${ }^{1}$ and Cesar Garita ${ }^{2}$ \\ ${ }^{1}$ TEC-Digital, \\ Costa Rica Institute of Technology (TEC), Cartago, Costa Rica \\ machaconeitcr.ac.cr \\ ${ }^{2}$ Computing Research Center, School of Computer Science, \\ Costa Rica Institute of Technology (TEC), Cartago, Costa Rica \\ cesaraitcr.ac.cr
}

\begin{abstract}
E-learning projects in many universities are focused on adapting or installing a software platform to upload teaching materials and sometimes to open discussion forums. However, it is totally possible to extend the learning management system (LMS) as a complete service platform for students and instructors including more advanced services. This paper shows the progressive integration of services and applications in TEC Digital as the open source e-learning platform of the Costa Rica Institute of Technology. This integration experience could be used as a case of study for other universities.
\end{abstract}

\section{Introduction}

In 2008, the Costa Rica Institute of Technology (TEC) started a project called TEC Digital to renew its LMS (Learning Management System) platform. The general objective of TEC Digital was to incorporate ICT in the development of teaching activities at TEC [1]. One of the main requirements then was that it should be open source [2]. Thus, the open source strategy that guides the architecture of TEC Digital, offers a high degree of extensibility which has led to the development of novel tools in the areas of instructional design, m-learning, adaptive learning, business intelligence, usability, and competence management, among others.

This paper shows the progressive integration of services and applications in the TEC Digital e-learning platform. This work could be used as case of study for other universities facing the problem of integrating e-learning technologies using OSS.

\section{Related Work}

During the last two decades, many papers can be found concerning the comparison and adoption of LMS in universities [3][4]. There are different justifications for adopting one LMS or the other, based on given technical criteria [5]. In particular, there are several studies about Free/Libre Open Source Software (FLOSS) adoption in public sector and in e-learning. For instance Rossi et al, in [6], enumerated several 
individual, technological, organizational and environmental factors to be considered in OSS adoption. Also, in [7] the FLOSS community presents a useful study of adoption models and myths around open source solutions. In [8], a comparative analysis is presented regarding FLOSS LMS, and one of the main conclusions is that despite technical functionalities, the final decision is greatly affected by end user needs.

In terms of open source LMS architecture approaches, there are several works focusing on extensibility and service integration. In [9], an extendable open source architecture of e-learning systems is presented, based on core components and optional extensions. In [10], a service-oriented architecture for LMS is discussed to support interoperability between LMS and different systems and databases. The architecture of TEC Digital follows these principles of extensibility and interoperability in order to support the development of novel components, as described in the next section.

\section{Integrating Services in TEC Digital}

The process of adaptation and integration of the full e-learning system at TEC has involved the following main tasks or stages:

(1) Adoption of .LRN LMS within the university.

(2) Integration of internal information sources into .LRN.

(3) Integration of complementary services and applications.

(4) Development of novel complementary components.

These tasks are briefly explained in the following subsections.

\subsection{Adopting .LRN}

In TEC, the adoption of the LMS was based on a comparison study between different platforms such as Moodle, Sakai and .LRN. In the end, .LRN was selected mostly due to its virtual community management, portal creation and interface design. The main issues compared against those LMS included: technical staff, interface design, adaptability to our internal organization, community use and development support. Once the LMS was chosen, a pilot plan was started involving a group of instructors from a few schools. Then, in 2009 .LRN was officially adopted as institutional platform and it has been consolidated through daily use.

\subsection{Integration of Internal Information Sources}

It is very common that universities have the LMS installed on one platform and the administrative and academic information located on other different platforms. In TEC case, the academic and administrative information systems are based on a Microsoft platform. The .LRN architecture is based on open source solutions including PostgreSQL, OpenACS, AOLServer. In order to integrate information services 
from the internal university sources (e.g. student admission and registration department), a service-oriented architecture was implemented. This integration architecture allowed the addition of several features and tools such as: automatic users account creation from registry database, student/instructor profiles, and communities matching the internal organization.

\subsection{Integration of Services and Applications}

The TEC academic community requires several services and applications offered by third parties to support teaching and regular activities. Some of those services are: Web2Project, Munin, Limesurvey, CmapServer, OJS, GIT, Dspace. The integration of these components has been mostly done using OpenLDAP and integrating some functions internally into the LMS.

\subsection{Development of Novel Components}

Besides integrating existing services and components, TEC Digital has the objective of complementing (improving) the LMS platform with some advanced functionalities or tools for students and teachers as well as university managers and collaborators. Some of the components developed "in-house" that have been successfully integrated with .LRN include: Instructional Design Generator (course planning), Mobile Course (app for course information access), and Learning Activities Manager (evaluation). Please notice that the adoption and integration of the services and components described in this section, demands a highly extensible system architecture, based on well-defined layers, web services and protocols.

Through the described services and components, TEC digital manages around 12125 students, 1026 instructors, 148 virtual communities, 4481 courses, and 8500 user accesses by day.

\section{Conclusions}

Some of the major benefits for our university generated by TEC Digital open source solutions include: reduced software license costs, seamless integration of university systems, capacity building in open source solutions and perhaps, the biggest benefit of OSS investment is that the organization is the "owner" of its products and solutions.

On the other hand, there are some considerations regarding the use of OSS that should not be underestimated: the use of OSS in an organization and in particular at universities, must be carefully planned in several dimensions; the technical skills required for technicians supporting OSS platform can be different than in other platforms; the use of OSS does not mean no investment, the organization must invest in support, adaptation, integration and others, and finally, the definition and coordination of the technical team can be quite complex. 


\section{References}

[1] Garita, J.A., Alpízar, I., Chacón-Rivas, M.: OpenACS/dotLRN integration with ITCR platform. In: 8th OpenACS/dotLRN Conference, Cartago, Costa Rica, pp. 27-35 (2009)

[2] Garita, C., Chacón-Rivas, M.: TEC Digital: A case study of an e-learning environment for higher education in Costa Rica. In: Proceedings of ITHET 2012. IEEE Proceedings ITHET, pp. 1-6 (2012)

[3] Ozkan, S., Koseler, R.: Multi-Dimensional Evaluation of E-Learning Systems in the Higher Education Context: An Empirical Investigation of a Computer Literacy Course. Presented at the 39th ASEE/IEEE Frontiers in Education Conference, pp. 1-6

[4] Aberdour, M.: Open Source Learning Management Systems. In: EPIC (2007)

[5] Moreno, P., Cerverón, V.: Plataforma tecnológica para potenciar los procesos de enseñanzaaprendizaje: desarrollo en la Universitat de València basado en software libre y colaborativo. In: Proc: SIIE 2006. VIII Simposio Internacional de Informática Aplicada a la Enseñanza (2006)

[6] Rossi, B., Russo, B., Succi, G.: Free/Libre Open Source Adoption in the Public Sector: Current State and Lessons Learnt

[7] FLOSSMetrics project, http://flossmetrics.org/ (accessed October 13, 2010)

[8] Fernandes, S., Cerone, A., Barbosa, L., Papadopoulos, P.: FLOSS in TechnologyEnhanced Learning,

http://mlab.csd.auth.gr/index $\cdot \mathrm{php} / \mathrm{gr} / \mathrm{publications?view=}$ publication\&task=show\&id=44 (accessed January 24, 2014)

[9] Khan, M.A., UrRehman, F.: An Extendable Open Source Architecture of e-Learning System

[10] Jabr, M.A., Omari, H.K.: E-learning management system using service oriented architecture. J. Comput. Sci. 6(3), 285 (2010) 